\title{
Large sporadic abdominal wall desmoid tumor due to repeated caesarean sections, a rare and long-term unexpected morbidity
}

\author{
Priti Agrawal $^{1 *}$, Rishi Agrawal ${ }^{2}$, Anandi Lobo ${ }^{3}$
}

\begin{abstract}
${ }^{1}$ Department of Obstetrics, Gynecology and Infertility, ${ }^{2}$ Department of General and Laparoscopic surgery, Aarogya Hospital and Test Tube Baby Center, Raipur, Chhattisgarh, India

${ }^{3}$ Department of Pathology, Aarogya Hospital and Test Tube Baby Center, Raipur, Chhattisgarh, India
\end{abstract}

Received: 09 October 2020

Accepted: 13 November 2020

\author{
*Correspondence: \\ Dr. Priti Agrawal, \\ E-mail: drpritiagrawal15@gmail.com
}

Copyright: () the author(s), publisher and licensee Medip Academy. This is an open-access article distributed under the terms of the Creative Commons Attribution Non-Commercial License, which permits unrestricted non-commercial use, distribution, and reproduction in any medium, provided the original work is properly cited.

\begin{abstract}
We present a case of large abdominal wall desmoid tumor (DT) arising due to repeated caesarean sections (CS) in a young woman of reproductive age group and managed with wide resection of tumor and abdominal wall reconstruction (AWR). Our patient was a 30-year-old female, came with complaints of persistent abdominal pain associated with mass on abdominal wall and difficulty in walking for 2 years. Patient had 3 living children, all were delivered by CS. Ultrasonography and CT scan of abdomen with contrast revealed a large solid abdominal wall mass and FNAC was suggestive of benign spindle cell neoplasm. We performed a wide surgical excision of tumor. Abdominal wall reconstruction done with prolene mesh. Post-operative period was uneventful with satisfactory wound healing. DTs are rare tumors that occur anywhere in the body and have quite variable clinical behavior. In our patient this tumor had occurred sporadically about 4-5 years of last child birth. Repeated trauma of surgeries on the abdominal wall led to the catastrophic destruction of the musculature in our patient. In this case CT scan revealed solid lump on abdominal wall without any evidence of incisional or inguinal hernia and associated intraabdominal pathology. Our specimen tested positive for $\beta$ catenin confirming DT. Surgery remains the main stay of treatment in all patients. In female patients presenting with lower abdominal wall tumor with history of previous CS or gynecological surgeries, DT should be considered.
\end{abstract}

Keywords: Caesarean sections, Cytology and GYN pathology, Gynecologic imaging

\section{INTRODUCTION}

Desmoid tumors (DT) are rare benign tumors exhibiting fibroblastic proliferation that typically arise from fascial or musculo aponeurotic structures. They account for $0.03 \%$ of all documented neoplasms and approximately $3 \%$ of all soft tissue tumors, with an incidence of 2-4 million per year. ${ }^{1}$

DT can occur sporadically or as a part of familial adenomatous polyposis coli (FAP) caused by adenomatous polyposis coli (APC) gene mutation.
Desmoid tumours of the abdominal wall may be associated with the surgical trauma of abdominal or pelvic surgery or physiologic trauma such as pregnancy. ${ }^{2}$ Given these associations, it unveils that DT peak at reproductive age, and are prone to occur after repeated caesarean sections (CS). These tumors have a propensity to infiltrate surrounding structures, rather than metastasize and have the potential to cause significant morbidity and mortality due to pressure effects and obstruction to vital organs.

Increased incidence occurring during and after pregnancy, following use of oral contraceptives, the preponderance of women of reproductive age, 
spontaneous tumor regression during menopause, expression of estrogen receptors and tumor regression with anti-estrogen treatment are all factors which indicate a potential etiological role of female sex hormones. ${ }^{3}$

We present a case of large abdominal wall DT arising due to repeated $\mathrm{CS}$ in a young woman of reproductive age group and managed with wide resection of tumor and abdominal wall reconstruction (AWR). In various case reports. ${ }^{1-3}$ DT occurred during pregnancy or after one CS in post-partum period. This is only case of the previous 3 CS scar on abdominal wall in a short span of six years which lead to large DT having mutilating effect on abdominal wall.

\section{CASE REPORT}

Our patient was a 30-year-old female, BMI-23.7 belonging to low socio-economic status and housewife came with complaints of persistent abdominal pain associated with mass on abdominal wall and difficulty in walking for 2 years. The pain was constant and dull aching aggravated by body movements. The size of the lump was gradually increasing. Patient had 3 living children, all were delivered by CS. During third CS tubectomy was also done. First two CS were done by midline vertical incision and third CS was done by Pfannenstiel incision. Her last child birth was 5 years back. On examination large lump about $15 \times 10 \times 10 \mathrm{~cm}$ occupying right side of midline scar and extending from right hypochondriac region superiorly and inferiorly up to right inguinal region, mobile, firm, with no cough impulse was felt.

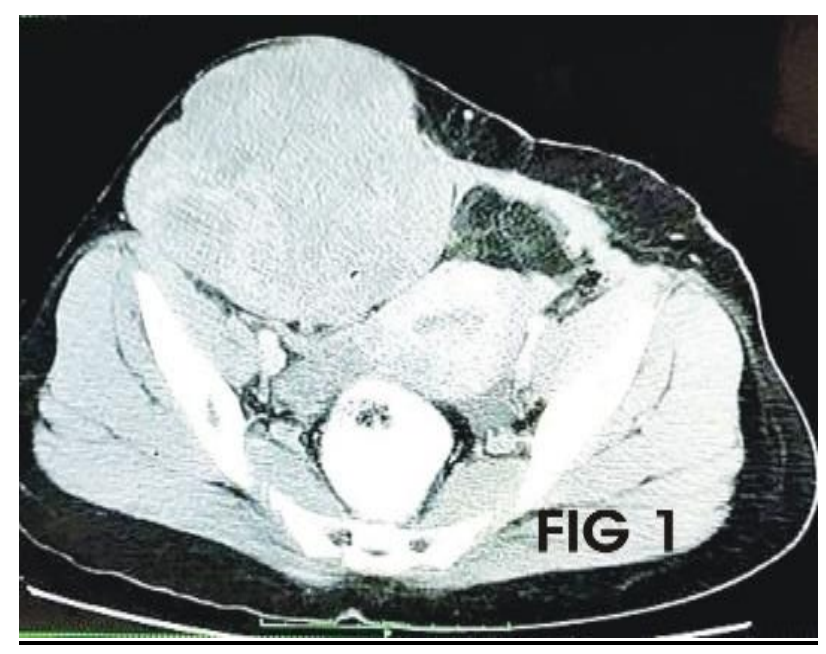

Figure 1: CT of lower abdomen showing desmoid tumour.

Ultrasonography and CT scan of abdomen with contrast revealed a large lobulated, roundish, soft tissue density mass showing temporally progressive, heterogeneous enhancement involving the right abdominal wall in umbilical and infra-umbilical location showing intraabdominopelvic protrusion measuring $12 \times 11 \times 12 \mathrm{~cm}$ with no other abdominal wall or intraabdominal abnormality (Figure 1).

FNAC was done and cytology smears revealed scattered fragments of benign spindle shaped cells, arranged in clusters along with single dispersed oval to spindle shaped cells in hemorrhagic background with no evidence of atypical or malignant cells, suggestive of benign spindle cell neoplasm.

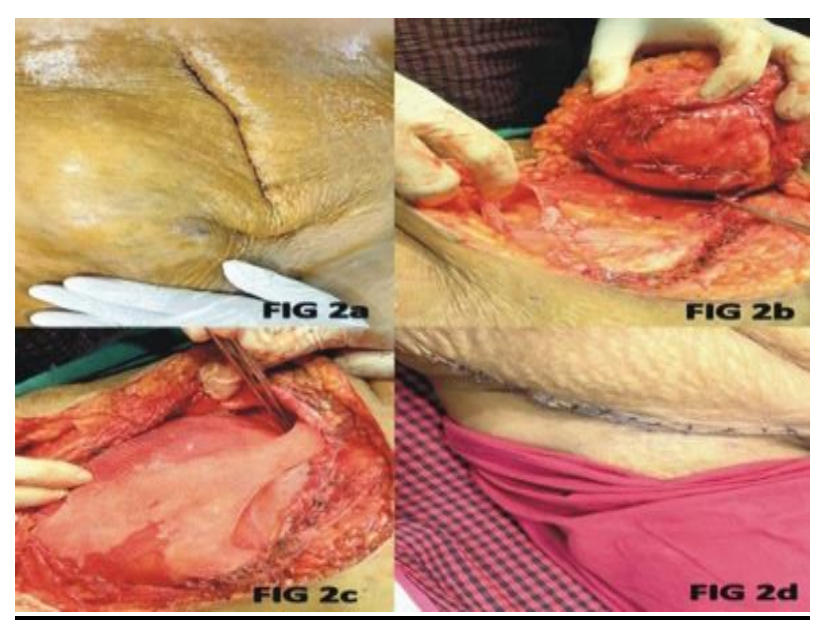

Figure 2: (a) Preoperative abdominal lump (b) intraoperative excision of lump.

(c) extraperitoneal mesh placement (d) wound healing on post-operative day 10.

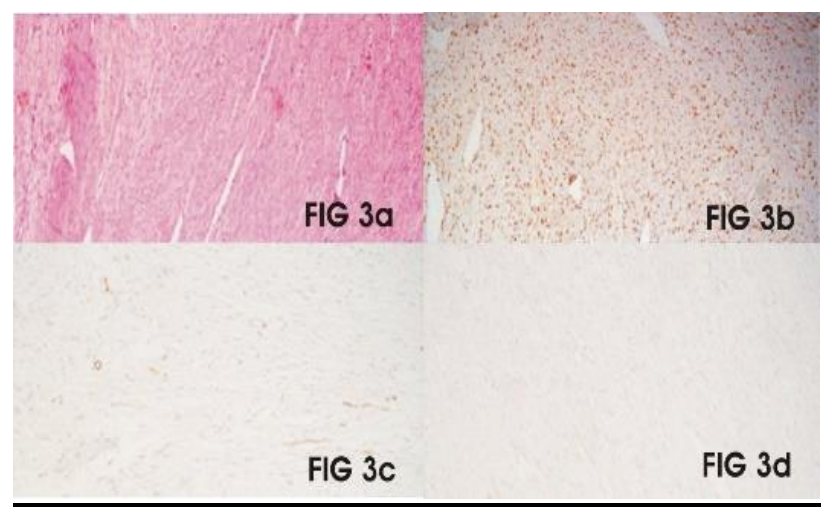

Figure 3: (a) H\&E; 200X) histopathological sections (b) smooth muscle actin (c) Desmin (d) 200X; IHC: strong nuclear positivity for $\beta$-catenin.

We performed a wide surgical excision of tumor measuring $16 \times 12 \times 12 \mathrm{~cm}$. Entire lump was extra peritoneal with no involvement of intraabdominal organs. Extraperitoneal prolene mesh $30 \times 30 \mathrm{~cm}$ placed and fixed to surrounding bony prominences and muscles with prolene 2-0 (Figure 2). Abdominal wall reconstruction done by mobilizing the muscles on left side and fixing to inguinal ligament, fascia lata and muscles of lateral abdominal wall on right side. Another prolene mesh $15 \times 7$ $\mathrm{cm}$ placed over the approximated muscles covering inguinal ligament. Drain was kept in situ which was removed on third postoperative day. Post-operative 
period was uneventful and patient was discharged on fifth postoperative day. Wound healing was satisfactory.

The excised tumor measured $16 \times 12 \times 12 \mathrm{~cm}$ with attached skin and subcutaneous tissue, cut surface revealed a well circumscribed solid, firm mass with no areas of hemorrhage or necrosis.

Histopathology of the specimen was suggestive of DT and immunohistochemistry (IHC) tested positive for $\beta$ catenin and negative for desmin (Figure 3).

\section{DISCUSSION}

DTs are rare tumors that occur anywhere in the body and have quite variable clinical behavior. The majority of DT arise sporadically while $5-15 \%$ are associated with FAP. ${ }^{4}$ The vast majority of these tumors are in the abdominal wall while intraabdominal tumors are rare.

In our patient this tumor had occurred about 4-5 years after last child birth, sporadically with no history FAP. The most common site is in the right rectus abdominus muscles resulting from stretching of the abdominal muscles fascia during gestation. Repeated trauma of surgeries on the abdominal wall led to the catastrophic destruction of the musculature in our patient. ${ }^{2}$ Surgery and inflammation may induce tumor growth. Typically, wound healing is a strictly controlled, self-limited process of tissue regeneration and remodeling. In response to tissue injury, mesenchymal cells are recruited to promote wound healing, these pluripotent cells differentiate into fibroblast, myofibroblast and endothelial cells. Therefore, the phenomenon of repair, cicatrization, correction and healing may lead to series of events causing DT. ${ }^{5}$

Besides trauma, hormonal stimulation due to repeated pregnancies might have attributed. The fact that some DT have rapid growth during pregnancy, and regress without treatment after termination of pregnancy indicate their hormonal association. Further these desmoids also respond to antiestrogen drugs like tamoxifen.

The abdominal wall masses present with pain in $80 \%$ patients, about $46.7 \%$ experience cyclic pain due to abdominal wall endometrioma (AWE). ${ }^{6}$ Our patient had constant pain not varying menstrual cycles.

The distribution of abdominal masses in study by Khamchian et al were AWE (63.3\%) abdominal wall tumor $(13.3 \%)$, incisional hernia $(10 \%$,$) suture$ granuloma $(6.7 \%)$, inguinal hernia $(3.3 \%)$ and DT (3.3\%). ${ }^{6}$ Rarely voluminous foreign body granuloma consequent to the spillage of meconium and vernixcaseosa during CS may develop in the abdominal wall mimicking DT. ${ }^{7}$

Diagnosis of abdominal wall masses needs workup with ultrasonography, computed tomography and FNAC. In this case CT scan revealed solid lump on abdominal wall without any evidence of incisional or inguinal hernia and associated intraabdominal pathology. CT scan is the most commonly used imaging modality for the diagnosis and follow up of the patients of DT. ${ }^{8}$

$\beta$ catenin expression in DT as evaluated by IHC has an overall prevalence ranging from 82 to $100 \%$. Negative results of such an assay would not preclude the diagnosis of DT. However, given that most desmoid harbor CTNNBI gene mutations, a positive result for such mutation would serve to confirm the diagnosis of DT. Our specimen tested positive for $\beta$ catenin confirming DT and suggesting high risk of recurrence in our patient. ${ }^{9}$

Our patient was already tubectomised otherwise a followup period of at least 2 years before planning a subsequent pregnancy may be advisable though pregnancy does not increase the local recurrence rate after surgical resection of desmoid type fibromatosis. ${ }^{10-11}$

Surgery remains the main stay of treatment in all patients with abdominal-wall DT, failing active surveillance. In addition to surgery other therapies such as anti-estrogens, chemotherapy and radiation have been proposed. ${ }^{12}$

Surgical resection and mesh repair of defect are wildly accepted treatment. Our patient came from rural area and was not ready for active surveillance and so we decided to excise the tumor with AWR. ${ }^{13}$ Because of the repeated child birth the abdominal wall was quite lax and so we could achieve approximation of skin easily. Other methods used in AWR for larger defects are latissimus dorsi myocutaneous flap, tensor fascia lata pedicled flap, myofascial flaps with microsurgical end to end anastomosis. Despite the need to remove extensive margins, approximation can be achieved in the majority of the procedures without need of complex reconstructions. Even a repeat $\mathrm{CS}$ is possible after desmoid resection and abdominal mesh repair. ${ }^{14}$ Mendenhall et al reviewed 10 year postoperative patients with surgery only and found that the local control rate of the negative margins (73\%) was significantly different from that of the positive $(46 \%) .{ }^{15}$ Thus it is of critical importance to achieve the wide resection margins to control the desmoid recurrence rate. We have done wide surgical resection of the tumor thereby reducing the chances of recurrence.

Our patient is a young female and will require a long term follow up to see the recurrence of tumor or complications arising from AWR and mesh.

\section{CONCLUSION}

In female patients presenting with lower abdominal wall tumor with history of previous CS or gynaecological surgeries DT should be considered. FNAC helps in preoperative but the definite diagnosis relies on histopathology. The treatment of the DT is the wide surgical excision and repair of the abdominal wall. These 
cases need close follow up due to the risk of recurrence. Subsequent pregnancy do not appear to be contradicted

Funding: No funding sources

Conflict of interest: None declared

Ethical approval: Not required

\section{REFERENCES}

1. Carneiro C, Hurtubis C, Singh M, Robinson W. Desmoid tumors of the right rectus abdominus muscle in postpartum women. Arch Gynecol Obstet. 2009;279:869-73.

2. Robinston WA, Mc Millan C, Kendall A, Pearlman N. Desmoid Tumors in pregnant and Postpartum Women. Cancers. 2012;4:184-92.

3. Leon MG, Moussa HN, Movahedian M, Viteri OA, Longo M, Sibai BM. A rapidly growing abdominal mass:Desmoid tumor in pregnancy. AJP Rep. 2015;5(1):14-7.

4. Nieuwenhuis M, Casparie M, Mathus Vliegen L, Dekker O, Hogendoom P, Vasen H. A nationwide study comparing sporadic and familial adenomatous polyposis related desmoid type fibromatoses. Int $\mathbf{J}$ Cancer. 2011;129:256-61.

5. Zhou H, Lu H, Wang L, Xie L, Wu M, Li J et al. Abdominal wall desmoid tumor during pregnancy: Case report and literature review. Clin Exp Obestet Gynecol. 2015,42(2):253-8.

6. Khamchian T, Alizargar J, Mazoachi T. 5-year data analysis of patient following abdominal-wall endometrioma surgery. BMC Women's Health. 2014;14(1):151.

7. Maccio A, Kotsonis P, Madeddu C, A PET-positive rapidly growing mass of the abdominal wall after cesarean section with an unexpected diagnosis of vernixcaseosa granuloma a case report. Gynecol. Surg. 2018,15:17.
8. Amirfarzan MD, Keraliya AR, Krajewsti KM, Tirumani SH, Shinagare AB, Hornick JL, et al. Role of Imaging in management of desmoid type fibromatosis: A Primer for Radiologist. Radiographic. 2016,36:767-78.

9. Lazar AJF, Tuvin T, Lev D, Specific mutation in the $\beta$ catenin gene (CTNNB1), Correlate with local Recurrence in sporadic Desmoid Tumors. Am J Pathol. 2008;173(5):1518-27.

10. Bree ED, Dimitriadis E, Glannikaki E, Chryssov EG, Melissas J. A giant pregnancy associated intraabdominal desmoid tumor: not necessarily a contraindication for subsequent pregnancy. World $\mathbf{J}$ surgical oncol. 2013;11:277.

11. Cates JMM. Pregnancy does not increase the local recurrence rate after surgical resection of desmoid type filromatosis. Int J Clin Oncol. 2015;20(3):61722.

12. The Desmoid Tumor Working Group. The management of desmoid tumors: A joint global consensus-based guideline approach for adult and pediatric patients: Review. 2020;127:96-107.

13. Couto Netto SD, Teixeira F, Menegozzo CAM, Albertini A, Akaishi EH, Utiyama EM. Abdominalwall reconstruction after desmoid type fibromatosis radical resection :Case series from single institution and review of the literature. Int J Surg case Rep. 2017;33:167-72.

14. Ooi S, Ngo H. caesarian section after abdominal mesh repair for pregnancy-related desmoid tumor, a case report. Int J Womens Health. 2017;9:497-9.

15. Mendenhall WM, Zlotecki RA, Morris CG, Hochwald SN, Scarborough MT. Aggressive fibromatosis. AM J Clin Onco. 2005;28:211-5

Cite this article as: Agrawal P, Agrawal R, Lobo A. Large sporadic abdominal wall desmoid tumor due to repeated caesarean sections, a rare and long-term unexpected morbidity. Int J Reprod Contracept Obstet Gynecol 2020;9:5193-6. 